\title{
De novo transcriptome sequencing and analysis of Hydrilla verticillata (L.f.) Royle
}

\author{
Etika Goyal $^{1 \|}$, Singh Amit Kumar ${ }^{1 \text { II }}$, Ravi Shankar Singh ${ }^{1 \# 2}$, Ajay Kumar Mahato ${ }^{1}$ and Kumar \\ Kanika $^{1^{*}}$
}

\author{
${ }^{1}$ Biotechnology and Climate Change Laboratory, ICAR-National Research Centre on Plant Biotechnology, New \\ Delhi, India

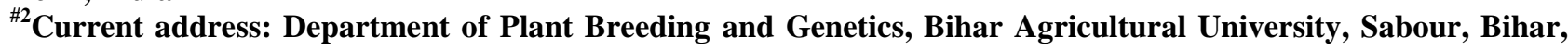 \\ India
}

*Corresponding author: kumarkanika@ rediffmail.com

II These authors contributed equally to this work.

\begin{abstract}
Hydrilla verticillata (L.f.) Royle, an aquatic plant, best documented example of an inducible $\mathrm{C}_{4}$ photosynthetic system, which concentrates $\mathrm{CO}_{2}$ in the chloroplasts without enzymatic compartmentation in mesophyll and bundle sheath cells. H. verticillata is a facultative $\mathrm{C}_{4}$ plant, which shifts from $\mathrm{C}_{3}$ to $\mathrm{C}_{4}$ photosynthesis under certain conditions, but lacks Kranz anatomy. Little is known about the molecular changes required for the transition to the $\mathrm{C}_{4}$ carbon concentrating mechanism (CCM). To gain insight into the processes that are involved in the $\mathrm{C}_{3}$ to the $\mathrm{C}_{4}$ transition, high throughput transcriptome sequencing and analysis were carried out using 454-GS FLX Titanium technology. A total of 533,595 reads were obtained after quality filtering. From these reads, 1,813 "Expressed sequence tags (ESTs)" were generated yielding 1,538 unigenes. Almost 95\% of these unigenes aligned to the "Nonredundant (nr)" database, whereas $4.88 \%$ did not show homology to any other known gene. "Reads per kilobase per million sequenced reads (RPKM)" analysis indicated that 283 unigenes were up-regulated and 383 unigenes were down-regulated in the $\mathrm{C}_{4}$ state as compared to the $\mathrm{C}_{3}$ state. A number of transcription factors found in our study were involved in photosynthesis. These consisted of, HAP3/NF-YB, AP/EREBP and $\mathrm{C} 2 \mathrm{H} 2$ family. GO, COG and KEGG analysis indicated that many genes were involved in energy metabolism, nucleotide metabolism, photosynthesis, signal transduction, stress responses and are worthy of further investigation. This transcriptome analysis is an important first step towards understanding the molecular changes underpinning the transition from the $\mathrm{C}_{3}$ to the $\mathrm{C}_{4}$ photosynthesis in $\mathrm{H}$. verticillata. It could also be a useful genomic resource for engineering $\mathrm{C}_{4}$ photosynthetic CCM in $\mathrm{C}_{3}$ crops.
\end{abstract}

Keywords: Hydrilla verticillata, Transcriptome, Next generation sequencing, 454 pyrosequencing.

Abbreviations: HvC, Hydrilla control sample; HvT, Hydrilla treated sample; COG, Clusters of Orthologous Groups; GO, Gene Ontology; SRA, Sequence Read Archive.

\section{Introduction}

$\mathrm{C}_{4}$ photosynthesis is a composite biological trait that enables plants to accumulate biomass at a much faster rate and thrive even in adverse environments compared to $\mathrm{C}_{3}$ plants (Brautigam et al., 2011). $\mathrm{C}_{3}$ plants such as rice (Oryza sativa L.), wheat (Triticum aestivum L.), soybean (Glycine max L.) and potato (Solanum tuberosum L.) assimilate atmospheric $\mathrm{CO}_{2}$ directly through the $\mathrm{C}_{3}$ photosynthetic pathway. $\mathrm{C}_{4}$ plants such as maize (Zea mays L.), sugarcane (Saccharum officinarum L.) and sorghum [(Sorghum bicolor L.) Moench] have a $\mathrm{C}_{4}$ photosynthetic pathway in addition to the $\mathrm{C}_{3}$ pathway (Miyao et al., 2011).

The most salient features of $\mathrm{C}_{4}$ plants are the presence of 'Kranz' anatomy and the cell-specific compartmentation of $\mathrm{C}_{4}$ enzymes (Hatch, 1988). Compartmentation, chloroplast differentiation and structural adaptations are considered prerequisites for $\mathrm{C}_{4}$ photosynthesis. In contrast, many aquatic autotrophs possess different modes of adaptation to cope with limited $\mathrm{CO}_{2}$ and high $\mathrm{O}_{2}$ conditions, including various $\mathrm{CO}_{2}$ concentrating mechanism (CCM) and the capacity to utilize $\mathrm{HCO}_{3}{ }^{-}$for photosynthesis (Bowes and Salvucci, 1989).
However, plants like Hydrilla verticillata and Borszczowia aralocaspica prove that 'Kranz' anatomy is not essential for $\mathrm{C}_{4}$ photosynthesis and that $\mathrm{C}_{4}$ metabolism can function even within single cell (Voznesenskaya et al., 2001; Edwards et al., 2004). Magnin et al., (1997) and Reiskind et al., (1989) established that, there is an intracellular compartmentalization of $\mathrm{C}_{4}$ and Calvin cycle (Reiskind et al., 1997). The carbon concentrating mechanisms were able to concentrate inorganic $\mathrm{CO}_{2}$ up to $400 \mathrm{mmol} \mathrm{m}^{-3}$ in the chloroplast.

$H$. verticillata is an aquatic macrophyte that can shift from $\mathrm{C}_{3}$ to $\mathrm{C}_{4}$ photosynthesis under low $\mathrm{CO}_{2}$ conditions, without its leaf cells undergoing any structural modifications (Rao et al., 2006a; Rao et al., 2002; Estavillo et al., 2007). During this transition, genes encoding $\mathrm{C}_{4}$-specific isozymes of phosphoenolpyruvate carboxylase (PEPC), pyruvate orthophosphate dikinase (PPDK), NADP-malic enzyme (NADP-ME) and carbonic anhydrase (CA) are up-regulated (Rao et al., 2006a; Rao et al., 2002; Estavillo et al., 2007; Brautigam et al., 2014; Reiskind and Maberly, 2014). In $H$. verticillata there are three isoforms of $P E P C$, of which only 
one isoform is up regulated in $\mathrm{C}_{4}$ leaves and has $\mathrm{C}_{4}$-like isoform kinetics (Rao et al., 2008). Two isoforms of PPDK and three of NADP-ME were also detected. One isoform of NADP-ME from the chloroplast was upregulated in light. This isoform had kinetic characteristics intermediate between $\mathrm{C}_{4}$ and $\mathrm{C}_{3}$ isoform of terrestrial plants (Estavillo et al., 2007; Rao et al., 2006b).

Higher temperatures and elevated $\mathrm{CO}_{2}$ level can reduce Rubisco activation (Kobza and Edwards, 1987; Sage, 1990; Brandner and Salvucci, 2000). In rice leaves rubisco deactivation triggered by increase in temperature is greater under higher $\mathrm{CO}_{2}$, conditions ( $\mathrm{Vu}$ et al., 1997). If the earth's surface temperature continues to increase with rising atmospheric $\mathrm{CO}_{2}$ there will be a need for agronomic crops with improved heat tolerance. There is a need for developing genotypes, suited to cope with global climate changes. $\mathrm{C}_{4}$ photosynthesis is advantageous when limitations on carbon acquisition are imposed, by high temperature, drought and saline conditions (Voznesenskaya et al., 2001). Further, $\mathrm{C}_{4}$ plants also have better nitrogen-use efficiency as compared to $\mathrm{C}_{3}$ plants, since the rate of photosynthesis per unit nitrogen in the leaf is increased as $\mathrm{C}_{4}$ plants need less of Rubisco enzyme. This enzyme works more efficiently under high $\mathrm{CO}_{2}$ concentrations in the bundle sheath cells unlike mesophyll cells in $\mathrm{C}_{3}$ plants. It is by far the most abundant protein in leaves of $\mathrm{C}_{3}$ plants (Oaks, 1994; Gowik and Westhoff, 2000). This has led to attempts to introduce components of $\mathrm{C}_{4}$ photosynthetic pathways in $\mathrm{C}_{3}$ crops, in order to increase productivity, especially under climate change scenario (Sheehy et al., 2000; Matsuoka et al., 2001). Introduction of all the characteristics of $\mathrm{C}_{4}$ photosynthesis to $\mathrm{C}_{3}$ crop plant like rice, will increase its photosynthetic potential and enable us to reap the net agronomic benefit in term of yield (Reynolds et al., 2010). An International Consortium is attempting to establish $\mathrm{C}_{4}$ pathway in rice (Furbank et al., 2009). To understand the complexity of the biochemical and anatomical traits necessary for this mechanism to operate is daunting and the minimal set of necessary genes is also unknown.

$H$. verticillata is a good system to decipher the minimal components required to run $\mathrm{C}_{4}$ photosynthesis without 'Kranz' anatomy. The inducible, single cell $\mathrm{C}_{4}$ system in $H$. verticillata is a minimalistic $\mathrm{C}_{4}$ model, which has led to different attempts to introduce a similar $\mathrm{C}_{4}$ pathway into the mesophyll cells of $\mathrm{C}_{3}$ cereals (Miyao, 2003; Hausler et al., 2002; Matsuoka et al., 2001; Miyao et al., 2011). This plant is a member of the hydrocharitaceae family and could provide insights into early events in the development and evolution of $\mathrm{C}_{4}$ photosynthesis (Rao et al., 2002).

Next generation sequencing (NGS) is a powerful tool, that sequences the whole genome and quantifies gene expression (Metzker, 2010). NGS enables de novo transcriptome sequencing and analysis of non-model species without annotated genomes. This has led to the expansion of databases related to various biological processes of even nonmodel species. The 454 GS FLX genome sequencer provides quarter million sequence reads of nearly $200-400$ bases in each run, from a cDNA template generated from mRNA (http://www.454.com/) (Metzker, 2010). These reads can be mapped onto a closely related reference to quantify the number of reads matching a gene locus, thereby providing a measure of transcript abundance (Flicek and Birney, 2009; Brautigam and Gowik, 2010).

In the present study, the transcriptome profile of $H$. verticillata during elevated temperature was generated using NGS technology. A number of genes/transcription factors that may be involved in the transition from the $\mathrm{C}_{3}$ to $\mathrm{C}_{4}$ photosynthetic pathway were putatively identified by de novo transcriptome analysis. Functional annotation and classification analysis of reads indicated that, many genes were related to photosynthesis, signal transduction, stress responses and various putative metabolic pathways.

\section{De novo transcriptome sequencing and assembly}

We followed the transition from the $\mathrm{C}_{3}$ to the $\mathrm{C}_{4}$ photosynthetic state prior to NGS, by monitoring the expression levels of the $H$. verticillata PEPC isoform-2 gene (PEPC-2) using semi-quantitative RT-PCR (Table S1). The expression level of $P E P C$-2 gene increased on day 8 sample kept at $34^{\circ} \mathrm{C}$. This increase in band intensity was confirmed by qRT-PCR using PEPC 2 gene specific primer (Fig. S1). Therefore, the day 8 sample kept on $34^{\circ} \mathrm{C}$ was used as the treatment (transition towards $\mathrm{C}_{4}$ state; HvT) and day 8 sample kept at $20^{\circ} \mathrm{C}$ was used as control (present in $\mathrm{C}_{3}$ state; $\mathrm{HvC}$ ). To develop a comprehensive understanding of the cues involved in the transition from the $\mathrm{C}_{3}$ to the $\mathrm{C}_{4}$ photosynthetic phase in $H$. verticillata, two cDNA libraries were prepared using mRNA samples from shoots of $\mathrm{HvC}$ (control) and HvT, respectively. Equimolar amounts of RNA from three biological replicates were pooled together prior to the cDNA synthesis. These samples were subjected to pair-end read (PE) sequencing using the Roche 454 sequencing platform. The sequencing run generated a total of 599,069 raw reads. After removal of adaptor sequences, a total of 533,595 high quality reads having 215,200,000 bp were obtained. An overview of the sequencing and assembly process is presented in Table 1. All the raw transcriptome data have been deposited at the Sequence Read Archive (SRA) of the National Centre for Biotechnology Information (NCBI) with the accession numbers SRR1300831 and SRR1300832for $\mathrm{HvC}$ and HvT, respectively. By using the Newbler assembler (V.2.6), a total of 1,831 ESTs were generated. Using Cd-hit (V.4.6.1) software (Li and Godzik, 2006), the ESTs were further assembled into 1,538 unigenes, with a minimum unigene length of 70bp, a maximum length of 5,300 bp and an average length of $993 \mathrm{bp}$. The size distribution of the unigenes is shown in Fig. S2A. Among these unigenes, 1,362 $(88.56 \%)$ were longer than $500 \mathrm{bp}$, and $494(36.27 \%)$ of these were longer than $1,000 \mathrm{bp}$. The GC contents of the putative genes were also examined as it can provide information about their evolution, structure (intron size and number), regulation and DNA stability. The average GC content of $H$. verticillata unigenes was $50.9 \%$, which is in the range of $\mathrm{GC}$ levels of coding sequences in monocots (5060\%) (Kuhl et al., 2004) (Fig. S2B).

\section{Gene sequence annotation and classification}

A sequence similarity search was conducted using Blast $2 \mathrm{GO}$ software against the NCBI nr protein database via Basic Local Alignment Search Tool (BLAST) algorithm specifying E-values $1.0 \mathrm{E}^{-3}$ followed by functional annotation (Conesa and Gotz, 2008; Gotz et al., 2008; Myhre et al., 2006). Among the assembled unigenes, 95.12\% (1,463 unigenes) were aligned to the nr database (Table S2). These assembled unigenes aligned mainly to Medicago truncatula (5.33\%), Magnetospirillum gryphiswaldense (3.28\%), Oryza sativa $(3.00 \%)$, Oxytricha trifallax $(6.83 \%)$ and Vitis vinifera (5.33\%) (Fig. 1).

GO classification revealed that, out of $1,463 \mathrm{nr}$ hits, a total of 786 unigenes were categorised into 27 functional groups (Fig. 2), including biological process, cellular component and molecular function. In total, 5,622 GO assignments were 
obtained, with 1,985 (35.31\%) for biological process, 1,720 $(30.59 \%)$ for cellular component and $1,043(18.55 \%)$ for molecular function at level 2. In the molecular function category, binding $(39.12 \%)$ and catalytic activity $(37.39 \%)$ were predominant. In the cellular component category, cell $(36.22 \%)$ and organelle $(31.51 \%)$ were the most prominent classes. In the biological process category, most of the unigenes were involved in metabolic processes (29\%) and cellular processes $(22.97 \%)$ (Table S3).

Finally, to evaluate the completeness of the $H$. verticillata transcriptome and the effectiveness of the annotation process, all the unigenes were submitted to the Clusters of Orthologous Groups (COG) database for functional prediction and classification. COG classification of 1,463 nr hits indicated that $1,394(95.28 \%)$ unigenes were clustered into 24 functional categories. Some unigenes had multiple COG functions, therefore, a total of 1,461 functional annotations were produced. Among the 24 functional categories, "energy production and conversion" (272, $18.62 \%$ ) represented the largest group, followed by "translation" (188, 12.87\%), "general functional prediction only" (186, 12.73\%), "post translational modification, protein turnover, chaperone function" $(134,9.17 \%)$, "Inorganic ion transport and metabolism" (104, 7.12\%), "carbohydrate metabolism and transport" $(93,6.37 \%)$ and "amino acid metabolism and transport" $(87,5.95 \%)$. The smallest groups were "RNA processing and modification" $(3,0.20 \%)$ and "defence mechanism" (9, 0.62\%) (Fig. 3).

KO (KEGG Orthology) numbers were assigned to 609 unigenes that comprised only two contigs and 607 isotigs, which were involved in 216 different pathways. A summary of the sequences involved in these pathways is described in Table S4. The six hundred and nine KEGG annotated unigenes were categorised into six different functional groups mainly "metabolism", "genetic information processing", "cellular process" and "environmental information processing" (Table 2). The pathways represented by the KEGG annotated unigenes were mostly involved in Ribosome (ko03010), Photosynthesis (ko00195), Oxidative phosphorylation (ko00190), Carbon metabolism (ko01200), Carbon fixation in photosynthetic organisms (ko00710), Biosynthesis of amino acid (ko01230) and Glycolysis/ Gluconeogenesis (ko00010) (Fig. 4, Table 2).

\section{Analysis of differentially expressed transcripts and identification of transcription factors}

To study the expression level of unigenes in $\mathrm{HvC}$ and HvT, the number of clean reads was compared between the libraries for each of the 1,538 assembled unigenes through RPKM analysis (Mortazavi et al., 2008). A total of 666 unigenes were differentially expressed between $\mathrm{HvC}$ and the HvT (Fig. 5). Out of 666 unigenes, 283 unigenes were upregulated in $\mathrm{HvT}$ and down regulated in $\mathrm{HvC}$ while 383 unigenes were down-regulated in $\mathrm{HvT}$ and upregulated in HvC (Table S5). Interestingly, of the 1,463 unigenes under investigation, 204 and 186 unigenes were uniquely expressed in $\mathrm{HvT}$ and $\mathrm{HvC}$, respectively. All the assembled unigenes were analysed for the presence of transcription factors using the PlantTFcat online tool (http://plantgrn.noble.org/ PlantTFcat). A total of 44 unigenes, representing $2.86 \%$ of the transcriptome, belonged to 18 putative transcription factor (TF) families (Table S6) and those are represented by "Hap3/NF-YB", "C2H2" and AP2-EREBP TF family. These transcription factors are reported to play role in abiotic stress tolerance and photosynthesis improvement (Saibo et al., 2009).

\section{In silico identification of SSRs markers}

All the unigenes were used to mine potential SSRs to develop SSR markers. In total, 1041 SSRs were identified by SSR Locator in $650(42.26 \%)$ unigenes of $H$. verticillata. Out of these unigenes, 170 unigenes contained more than one SSR (Table 3). Hexa-nucleotide repeats $(77.33 \%)$ were the most abundant SSRs in the transcriptome. The second major class was tri-nucleotide $(14.32 \%)$ and the remaining repeat motifs were mononucleotide $(0.38 \%)$, dinucleotide (1.83\%), tetranucleotide $(5.28 \%)$ and pentanucleotide $(0.86 \%)$ (Fig. $6 a, 6 b)$.

SSRs with two tandem repeats $(76.37 \%)$ were the most common, followed by four, three, five and six tandem repeats. The dominant repeat motif in all the SSRs was AAGCGA/TCGCTT (33, 3.17\%), followed by AAAATC/GATTTT $(15,1.44 \%)$, CTC/GAG $(15,1.44 \%)$, CCG/CGG $(13,1.25 \%)$ and GGA/TCC $(12,1.15 \%)$ (Table S7). The analysis of GC content of microsatellites indicated that most of the SSRs were rich in GC content, except for mono- and penta- nucleotide repeats (Fig. 6c). Focusing on tri- and hexa- nucleotide repeats, SSRs with 50\%-100\% GC content were twice as prevalent as those with only $0-50 \%$ GC content. These SSRs can be used as functional markers in various genetic studies in the future. To make these SSR markers useful, we employed Primer 3 to design primer pairs for each SSR. In total, 872 primer pairs for 1,041 (83.77\%) SSRs were designed from the microsatellites with sufficient flanking sequences (Table S8).

\section{Discussion}

$H$. verticillata, an aquatic plant, is the best documented example of an inducible $\mathrm{C}_{4}$ photosynthetic system that concentrates $\mathrm{CO}_{2}$ in the chloroplasts without enzymatic compartmentation in mesophyll and bundle sheath cells. This plant lacks 'Kranz' anatomy and is a facultative $\mathrm{C}_{4}$ plant that shifts from $\mathrm{C}_{3}$ to $\mathrm{C}_{4}$ photosynthesis under certain conditions. The primitive types of $\mathrm{C}_{4}$ photosynthetic system without 'Kranz' anatomy in $H$. verticillata and Borszczowia aralocaspia (Chenopodiaceae) suggests that $\mathrm{C}_{4}$ photosynthetic system could run in single mesophyll cells (Freitag and Stichler, 2000; Magnin et al., 1997). Information at the molecular level related to the transition from the $\mathrm{C}_{3}$ photosynthetic pathway to the $\mathrm{C}_{4}$ photosynthetic pathway is not available. To gain insight into the processes that are involved in the transition from the $\mathrm{C}_{3}$ mode to the $\mathrm{C}_{4}$ mode of photosynthesis in $H$. verticillata, high throughput transcriptome sequencing and analysis was carried out in this study using 454-GS FLX Titanium technology.

Inducible photosynthetic system of $H$. verticillata provides an excellent opportunity to study the minimum essential biochemical elements required to operate a $\mathrm{C}_{4}$ photosynthetic system. Exceptionally all the enzymes and metabolite transporters involved in the $\mathrm{C}_{4}$ pathway also occur in $\mathrm{C}_{3}$ plants, although at much lower activities and having different tissue specificities (Hausler et al., 2002). Therefore, the introduction of individual $\mathrm{C}_{4}$ cycle genes might perturb the primary metabolism, and also in certain cases secondary metabolism (Hausler et al., 2002). Advent of genetic engineering in crop plants has raised hope for introduction of $\mathrm{C}_{4}$ characteristics in $\mathrm{C}_{3}$ plants by genetic manipulation of rice, tobacco and potato (Matsuoka et al., 2001). The facultative nature of $H$. verticillata also enables us to study the genes involved both in the $\mathrm{C}_{3}$ and the $\mathrm{C}_{4}$ states and to identify the differences in the expression of genes and 
Table 1. Summary of 454 transcriptome sequencing and assembly for H. verticillata.

\begin{tabular}{llll}
\hline Item & Library & Number & Total Bases $(\mathrm{Mb})$ \\
Raw reads & HvC & 284,588 & 112.8 \\
\hline & HvT & 314,481 & 123.5 \\
Clean reads & HvC & 250,969 & 101.6 \\
& HvT & 282,626 & 113.6 \\
Contigs & & & \\
No. of Contigs (n) & & 71 & - \\
Average Length (bp) & $1,190.76$ & - \\
Maximum Length (bp) & 4,177 & - \\
Minimum Length (bp) & & 516 & - \\
Singletons & & & \\
No. of Singletons (n) & & 1,467 & - \\
Average Length (bp) & & 983.23 & - \\
Maximum Length (bp) & & 5,300 & - \\
Minimum Length (bp) & & 70 & - \\
\hline
\end{tabular}

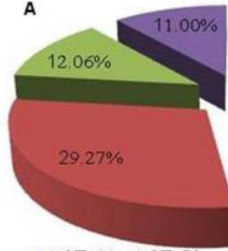

$=1.0 \mathrm{E}-4$ to $1.0 \mathrm{E}-50$

$=1.0 \mathrm{E}-100$ to $1.0 \mathrm{E}-150$

c

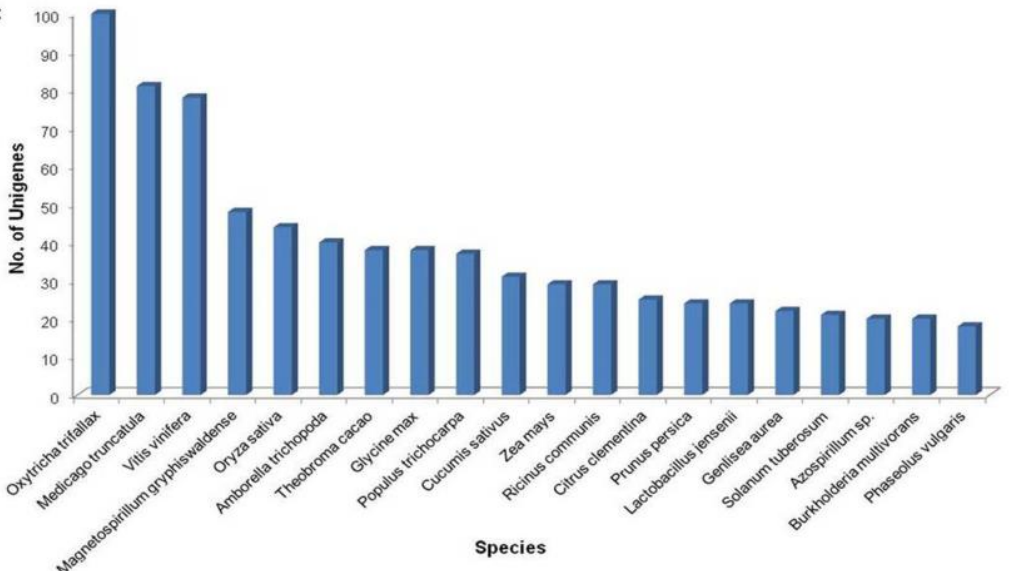

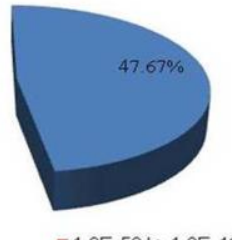

$=1.0 \mathrm{E}-50$ to $1.0 \mathrm{E}-100$

m $>1.0 \mathrm{E}-150$

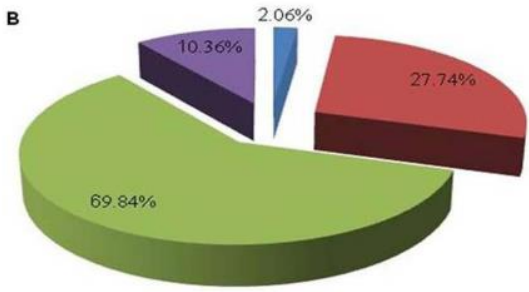

$=100 \%=90 \%$ to $100 \%=60 \%$ to $90 \%=$ less than $60 \%$

Fig 1. Characteristics of the homology search of the H. verticillata transition phase unigenes. (a) E-value distribution of the BLASTX hits against the $\mathrm{nr}$ protein database for each unigene, using an E-value cut-off of $10 \mathrm{E}^{-3}$; (b) Similarity distribution of the top BLAST hits for each unigene; (c) Number of unigenes matching the top 20 species using BLASTX.

variations in the regulatory and catalytic domains of the protein products.

One of the three primary carboxylases initiating the $\mathrm{C}_{4}$ CCM in H. verticillata PEPC isoform 2 is differentially expressed in $\mathrm{C}_{4}$ tissue (Rao et al., 2002; Magnin et al., 1997). In the present study, the expression level of the $H$. verticillata $P E P C-2$ expressed in HvT was monitored to follow the transition from the $\mathrm{C}_{3}$ to the $\mathrm{C}_{4}$ photosynthetic state prior to NGS. The PEPC-2 gene transcript level had exhibited an increase in expression on day 8 indicating an induction of the $\mathrm{C}_{4} \mathrm{CCM}$ under modified growth conditions. We specifically harvested samples kept at $34^{\circ} \mathrm{C}$ on day 8 (HvT) (Fig. S1).

To develop a comprehensive understanding of the cues involved in the transition from the $\mathrm{C}_{3}$ to $\mathrm{C}_{4}$ photosynthetic phase in $H$. verticillata, two cDNA libraries $\mathrm{HvC}$ and $\mathrm{HvT}$ were prepared. The pair-end read (PE) sequencing generated a total of 599,069 raw reads and a total of 533,595 high quality reads $(215,200,000 \mathrm{bp})$. From these reads a total of
1,831 ESTs and 1,538 unigenes, were obtained. Among the assembled unigenes, $95.12 \%$ (1,463 unigenes) aligned to the $\mathrm{nr}$ database (Table S2). Among these aligned unigenes in $\mathrm{nr}$ protein database, $52.33 \%$ of these genes indicated high homology with the gene sequences in the database, having Evalues less than $1.0 \mathrm{E}^{-50}$. The remaining $47.67 \%$ had an $\mathrm{E}$ value between $1.0 \mathrm{E}^{-4}$ to $1.0 \mathrm{E}^{-50}$ (Fig. 1a). The similarity distribution showed that $42.76 \%$ of the aligned unigenes had a similarity value higher than $85 \%$, whereas $10.36 \%$ of these genes had a similarity value lower than $60 \%$ (Fig. 1b). For species distribution, approximately $23.99 \%$ of the total unigenes matched with sequences from the five top-hit species, i.e., Medicago truncatula, Magnetospirillum gryphiswaldense, Oryza sativa, Oxytricha trifallax and Vitis vinifera. The twenty top-hit species based on $\mathrm{nr}$ annotation are shown in Fig. 1c. Among the unique sequences derived from these unigenes, coding sequences exhibited homology to sequences for proteins such as "cytochrome P450", "transcript antisense to rRNA protein", "cell wall associated 
Table 2. KEGG classification of $H$. verticillata unigenes.

\begin{tabular}{llll}
\hline KEGG categories & No. of unigenes & KEGG categories & No. of unigenes \\
\hline Metabolism & 386 & Environmental Information & 53 \\
& & Processing & 109 \\
Carbohydrate Metabolism & 111 & Signal Transduction & 102 \\
Energy Metabolism & 232 & Organismal System & 28 \\
Lipid Metabolism & 20 & Immune System & 49 \\
Nucleotide Metabolism & 12 & Endocrine System & 22 \\
Amino Acid Metabolism & 52 & Circulatory System & 13 \\
Metabolism of other Amino Acids & 9 & Digestive System & 7 \\
Metabolism of Cofactors and Vitamins & 15 & Excretory System & 35 \\
Metabolism of Terpenoids\& & 8 & Nervous System & 22 \\
Polyketides & & & 6 \\
Biosynthesis of other Secondary & 13 & Sensory System & \\
Metabolites & 27 & Development & 17 \\
Xenobiotics Degradation \& & & & 209 \\
Metabolism & 109 & Environmental Adaption & 39 \\
Genetic Information Processing & 11 & Human Disease & 15 \\
Transcription & 109 & Cancer & 114 \\
Translation & 27 & Immune Disease & 21 \\
Folding, Sorting \& Degradation & 3 & Neurodegenerative Disease & 21 \\
Replication \& Repair & 50 & Substance Dependence & 30 \\
Cellular Process & 40 & Circulatory Disease & 136 \\
Transport \& Catabolism & 25 & Endocrine \&Metabolic Disease & 609 \\
Cell Growth \& Death & 36 & Infectious Disease & \\
Cell Communication & & Total & \\
& & & \\
\hline
\end{tabular}

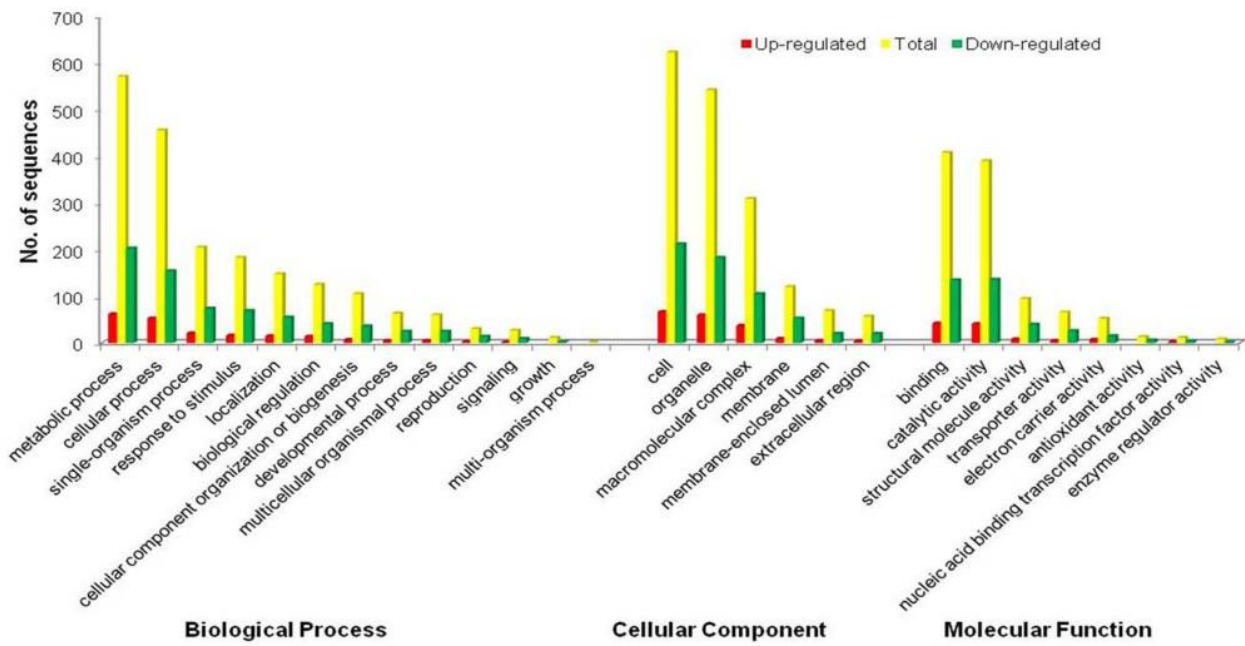

Fig 2. Gene Ontology classification of $H$. verticillata transcriptome and identification of differentially expressed genes in the $\mathrm{C}_{3}$ and $\mathrm{C}_{4}$ photosynthetic pathway. The $\mathrm{Y}$-axis represents number of unigenes and the $\mathrm{X}$-axis shows the $\mathrm{GO}$ categories.

hydrolase", "senescence protein", "amino transferase II", "thiazol biosynthesis enzyme", "Zinc finger", "serine hydroxyl methyl transferase", "NADH dehydrogenase", "ascorbate peroxidase esterase lipase", "tpr domain" and "chaperone protein chloroplastic like", etc. In the present study, the above mentioned putative functional transcripts that were identified can provide leads for future investigations.

\section{Functional annotation}

GO analysis provides a controlled vocabulary for the dynamic and hierarchical relationships between biological processes, cellular components and molecular functions, allowing a coherent annotation of gene products (Harris et al., 2004). In GO "biological process", includes "metabolic process", "cellular process", "response to stimulus", "singleorganism process", "localization" and "biological regulation". "Cellular process" comprises of "cell", "organelle", "macromolecular complex", "membrane", "extracellular region" and "membrane-enclosed lumen". "Molecular function" represented by "binding", "catalytic activity", "structural molecule activity", "transporter activity", "electron carrier activity" and "antioxidant activity" (Fig. 2).

COG is a database where orthologous gene products are classified (Tatusov et al., 2000). In the present transcriptome analysis, all the unigenes obtained represented 24 functional categories in the COG database (Fig. 3). The "energy production and conversion" represented the largest group, 
Table 3. Statistics of SSRs in the $H$. verticillata transcriptome.

\begin{tabular}{ll}
\hline Items & Numbers \\
\hline Total no. of SSRs & 1,041 \\
No. of SSR containing sequences & $650(42.26 \%)$ \\
No. of sequences containing more than one SSR & 170 \\
No. of mono-nucleotide SSR & $4(0.38 \%)$ \\
No. of di-nucleotide SSR & $19(1.83 \%)$ \\
No. of tri-nucleotide SSR & $149(14.32 \%)$ \\
No. of tetra-nucleotide SSR & $55(5.28 \%)$ \\
No. of penta-nucleotide SSR & $9(0.86 \%)$ \\
No. of hexa-nucleotide SSR & $805(77.33 \%)$ \\
\hline
\end{tabular}

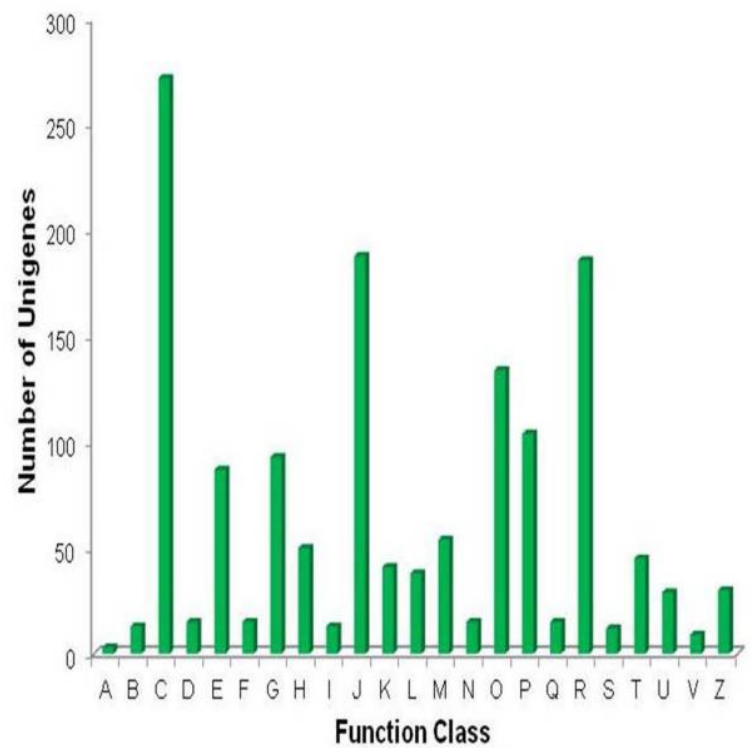

\author{
A: RNA processing \\ B: Chromatin structure \& dynamics \\ C: Energy production \& conversion \\ D: Cell cycle control \& mitosis \\ E: Amino acid metabolism \& transport \\ F: Nucleotide metabolism \& transport \\ G: Carbohydrate metabolism \& transport \\ H: Coenzyme metabolism \\ I: Lipid metabolism \\ $\mathrm{J}$ : Translation \\ $\mathrm{K}$ : Transcription \\ L: DNA Replication \& repai \\ M: Cell wall/ Membrane/ Envelope biogenesis \\ $\mathrm{N}$ : Cell motility \\ O: Posttranslational modification, protein turnover, chaperone \\ function \\ P: Inorganic ion transport \& metabolism \\ Q: Secondary structure \\ R: General function prediction only \\ S: Function unknown \\ T: Signal transduction \\ U: Intracellular trafficking \& secretion \\ Y: Nuclear structure \\ V: Defense mechanism \\ Z: Cytoskeleton
}

Fig 3. COG function classification of $H$. verticillata transcriptome. Graphical representation of clusters of orthologous (COG) classification. The number of unigenes is reported on the $\mathrm{Y}$-axis.

followed by "translation", "general functional prediction only", "post translational modification, protein turnover, chaperone function", "Inorganic ion transport and metabolism", "carbohydrate metabolism and transport" and "amino acid metabolism and transport". The smallest groups were "RNA processing and modification" and "defence mechanism. This result indicated that most of the unigenes were involved in "energy production and conversion", "carbohydrate metabolism and conversion" which probably evolved because of high consumption rate of energy. But detailed studies are required to prove this hypothesis.

In addition to GO analysis, KEGG pathway mapping based on enzyme commission (EC) numbers for assignments was also carried out for the assembled sequences. This alternative approach to categorise gene functions emphasises biochemical pathways. KEGG annotated unigenes were classified into six different functional groups. Following these analyses, the largest group, with $42.46 \%$ of the unigenes, was classified into "metabolism", with most of these genes involved in "carbohydrate metabolism", "nucleotide metabolism" and "energy metabolism". The results suggest that carbohydrate, energy and nucleotide metabolism were active during the transition between these photosynthesis modes. Sequences classified into "genetic information processing (GIP)" accounted for $12.00 \%$ of the KEGG annotated sequences. Well-represented metabolic pathways included "folding, sorting and degradation", "replication and repair", "translation" and "transcription". "Cellular processes" represented $5.50 \%$ of the KEGG annotated sequences. Processes such as "transport and catabolism", "cell growth and death" and "cell communication" were well represented. Additionally, 5.83\% of the sequences were classified into "environmental information processing (EIP)", for example, "signal transduction". "Organismal system" and "human disease" accounted for $11.22 \%$ and $22.99 \%$ of the unigenes, respectively (Fig. 4, Table 2). COG and KEGG pathway analysis are very useful techniques for predicting potential genes and the functions of these genes at a whole transcriptome level. The predicted molecular pathways, together with COG analysis, are useful for further investigations of the functions of genes.

Transcription factors (TFs) contain DNA-binding domains (DBDs) and regulate gene expression by binding to specific DNA sequences. TFs play important role in regulating growth, development and response to environmental factors by controlling gene variation. For example, Zhang et al., (2008) reported that ABP-9, a bZIP type TF has a role in improving the adaptability and stability of the photosynthetic apparatus to abiotic stress. They suggested that this TF potentially is related to expression of novel mechanism activated under water deficit and heat stress. In the present transcriptome profiling, a total of 18 putative transcription factors (TF) families were identified (Table S6). Among these, 11 unigenes represented the "Hap3/NF-YB" transcription factor, which were implicated in diverse developmental process. Further, the 2 unigenes represents AP2-EREBP and one unigene falls under $\mathrm{C} 2 \mathrm{H} 2$ transcription factor family, which plays role in photosynthesis under stress condition (Saibo et al., 2009). HAP3 genes were shown to 
Table 4. GO enrichment analysis of differentially expressed genes.

\begin{tabular}{|c|c|c|c|c|c|c|}
\hline GO-ID & Term & Category & $\begin{array}{l}\text { No. of differentially } \\
\text { expressed genes }\end{array}$ & $\begin{array}{l}\text { No. of unigenes } \\
\text { in subgroup }\end{array}$ & P-value & FDR \\
\hline \multicolumn{7}{|c|}{ Up-regulated in $\mathrm{C}_{4}$ photosynthetic pathway } \\
\hline Total & & & 128 & 1293 & & \\
\hline GO:0006950 & Response to stress & $\mathrm{P}$ & 5 & 131 & $9.56 \mathrm{E}-04$ & $6.60 \mathrm{E}-02$ \\
\hline GO:0006091 & $\begin{array}{l}\text { Generation of precursor } \\
\text { Metabolites and energy }\end{array}$ & $\mathrm{P}$ & 34 & 158 & $2.60 \mathrm{E}-03$ & $1.22 \mathrm{E}-01$ \\
\hline GO:0004518 & Nuclease activity & $\mathrm{F}$ & 7 & 12 & $3.26 \mathrm{E}-03$ & $1.22 \mathrm{E}-01$ \\
\hline GO:0003676 & Nucleic acid binding & $\mathrm{F}$ & 5 & 97 & $2.88 \mathrm{E}-02$ & $3.46 \mathrm{E}-01$ \\
\hline GO:0016788 & $\begin{array}{l}\text { Hydrolase activity, acting on } \\
\text { ester bonds }\end{array}$ & $\mathrm{F}$ & 7 & 15 & $8.26 \mathrm{E}-03$ & $1.42 \mathrm{E}-01$ \\
\hline \multicolumn{7}{|c|}{ Up-regulated in $\mathrm{C}_{3}$ photosynthetic pathway } \\
\hline Total & & & 484 & 810 & & \\
\hline GO:0009579 & Thylakoid & $\mathrm{C}$ & 35 & 114 & $2.84 \mathrm{E}-03$ & $1.95 \mathrm{E}-01$ \\
\hline GO:0006464 & $\begin{array}{l}\text { Cellular protein modification } \\
\text { process }\end{array}$ & $\mathrm{P}$ & 16 & 63 & 8.09E-03 & $1.95 \mathrm{E}-01$ \\
\hline GO:0016301 & Kinase activity & $\mathrm{F}$ & 13 & 8 & $8.95 \mathrm{E}-03$ & $1.95 \mathrm{E}-01$ \\
\hline GO:0009605 & Response to external stimulus & $\mathrm{P}$ & 10 & 5 & $1.09 \mathrm{E}-02$ & $1.95 \mathrm{E}-01$ \\
\hline GO:0006412 & translation & $\mathrm{P}$ & 48 & 63 & $3.00 \mathrm{E}-02$ & $3.62 \mathrm{E}-01$ \\
\hline GO:0016788 & $\begin{array}{l}\text { Hydrolase activity, acting on } \\
\text { ester bonds }\end{array}$ & $\mathrm{F}$ & 3 & 19 & 4.20E-02 & $3.71 \mathrm{E}-01$ \\
\hline GO:0005975 & $\begin{array}{l}\text { Carbohydrate metabolic } \\
\text { process }\end{array}$ & $\mathrm{P}$ & 44 & 58 & 4.30E-02 & $3.71 \mathrm{E}-01$ \\
\hline
\end{tabular}

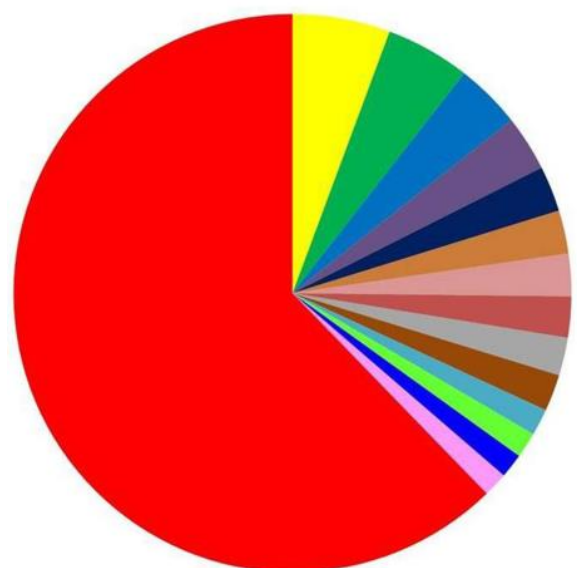

-Ribosome
- Photosynthesis
- Oxidative phosphorylation
- Carbon metabolism
- Parkinson's disease
- Photosynthesis - antenna proteins
" Carbon fixation in photosynthetic organisms
" Alzheimer's disease
" Huntington's disease
- Biosynthesis of amino acids
" Non-alcoholic fatty liver disease (NAFLD)
" Glycolysis / Gluconeogenesis
- Glyoxylate and dicarboxylate metabolism
" Phagosome
- Others

Fig 4. KEGG biochemical mappings of $H$. verticillata.

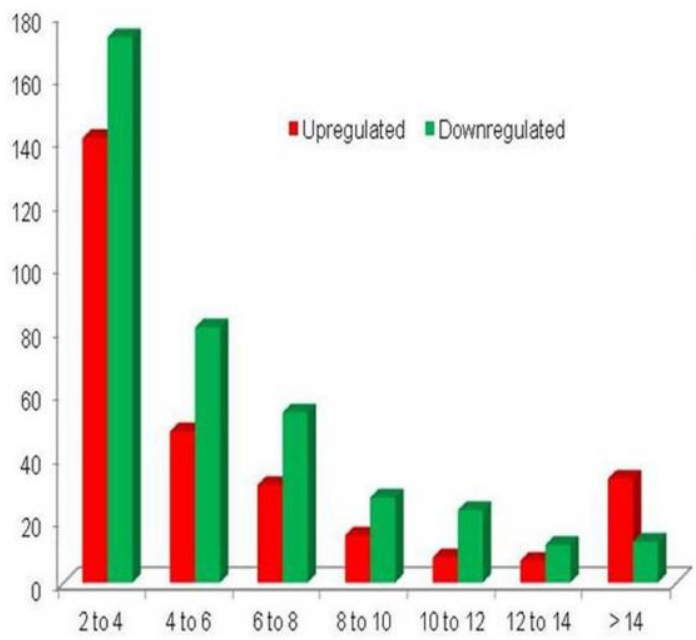

Fig 5. Overview of the differences in genes expression between the $\mathrm{C}_{3}$ and $\mathrm{C}_{4}$ photosynthetic pathway in $\mathrm{H}$. verticillata. Fold change distribution of the differentially expressed genes. 


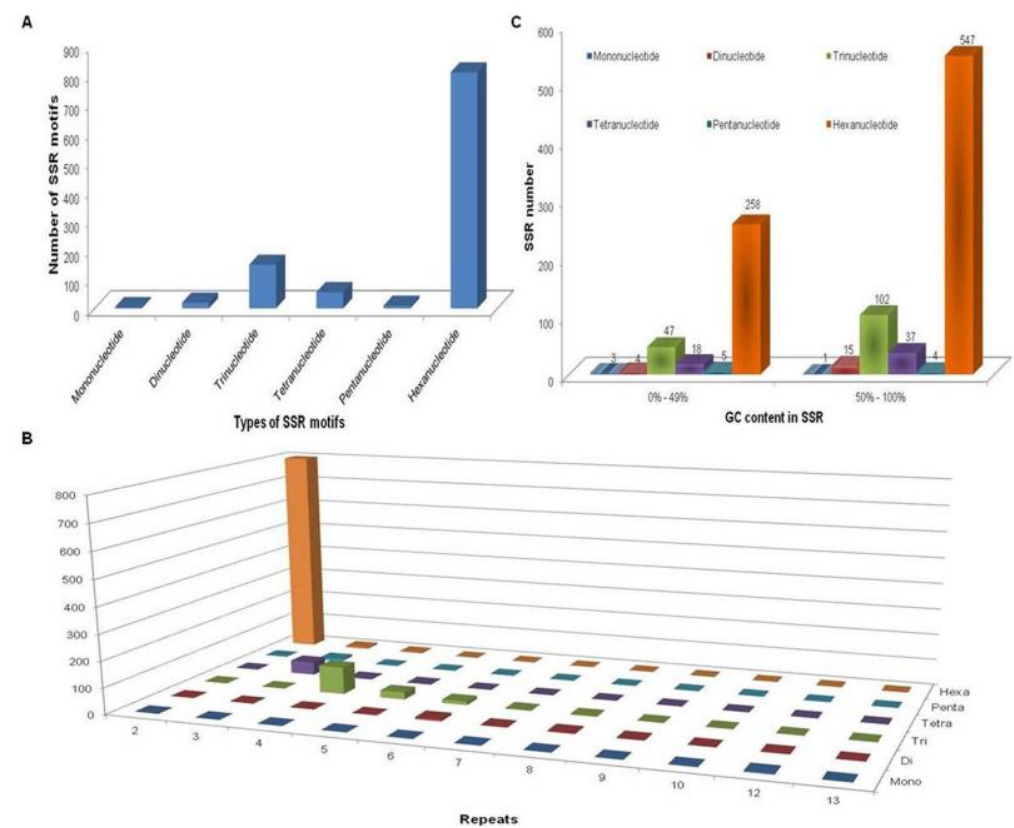

Fig 6. Distribution of SSRs. (a) Number of SSRs classified based on motif type; (b) GC content in microsatellites among different nucleotide types found in the transcriptome of H. verticillata; (c) Motif type and their frequency plotted as a function of the repeat number.

regulate the expression of nuclear-encoded chloroplast targeted genes and normal development of chloroplast in rice (Miyoshi et al., 2003). This Hap3/NF-YB" TF might be one of the candidates involved in the induction of photosynthetic genes. Park et al., (2001) and Guo et al., (2004) studied that AP2-EREBP TF lowers the loss of chlorophyll contents under high salt stress in tobacco. Hsieh et al., (2002) and Oh et al., (2007) observed that AP2-EREBP TF improves maximum quantum efficiency of PSII/ chlorophyll accumulation under different abiotic stresses. Kim et al., (2001) reported that $\mathrm{C} 2 \mathrm{H} 2 \mathrm{TF}$ helps in faster recovery of chlorophyll content under cold stress.

RPKM based comparative expression profiling study (Mortazavi et al., 2008) was done to systematically characterize the mRNAs present in $\mathrm{C}_{3}$ and $\mathrm{C}_{4}$ state, and also to identify the transcripts of differentially expressed genes. Six hundred sixty six (666) unigenes were differentially expressed (Table S5). The photosynthesis related genes (unigenes 46, 6.91\%) showed an over fourteen-fold change in expression level, which mainly included "photosystem I p750 chlorophyll a apoprotein al", "atp synthase subunit beta", "cell wall associated hydrolase" and "1-cys peroxiredoxin", while 314 unigenes $(47.15 \%)$ showed a 2 4-fold change, including "malate dehydrogenase" and "phosphoenolpyruvate carboxylase" (Fig. 5). These are the major $\mathrm{C}_{4}$ photosynthetic genes, which show that our samples have started to shift to the $\mathrm{C}_{4}$ photosynthetic pathway. Interestingly, of the 1,463 unigenes under investigation, 204 and 186 unigenes were uniquely expressed in $\mathrm{HvT}$ and $\mathrm{HvC}$, respectively.

\section{GO enrichment analysis of differentially expressed genes}

To study the function of differentially expressed genes, GO terms were extracted using the Blast2GO tool and subjected to GO enrichment analysis. Annotation of the differentially expressed genes revealed that 356 unigenes belonged to 26 GO groups, while the remaining 310 unigenes could not be classified (Fig. 2). The number of up-regulated unigenes (90) with GO annotations in HvT was less than the number of down-regulated unigenes (266). GO annotation revealed that many differentially expressed genes were involved in "transferase activity", "kinase activity", "response to stimulus", "biological regulation", "antioxidant activity" and "electron carrier activity". A majority of the up-regulated unigenes in HvT were involved in "nuclease activity", "thylakoid", "generation of precursor metabolites and energy" and "hydrolase activity". In contrast, the GO groups "cellular protein modification process", "protein modification process" and "DNA binding" were under-represented in the up-regulated unigenes of the $\mathrm{HvC}$ (Table 4).

\section{Marker development}

Molecular markers play an important role in plant biology. These are very commonly used for the analysis of plant genomes and identification of the association between genomic variation and heritable traits. SSRs (microsatellites) markers are usually associated with phenotypic and functional variations, so they can be utilized in marker assisted selection programmes based studies in plants. These SSRs are multi allelic in nature, reproducible, highly abundant. They cover the genome extensively and are codominant in nature (Wei et al., 2011; Powell et al., 1996). SSRs derived from ESTs, genes or cDNA clones are referred to as EST- or genic SSRs. Genic SSRs have some intrinsic advantages over genomic SSRs, such as ease of generation, stability and cross-species transferability. Genic SSRs are used in variety of studies, such as genetic mapping and assessment of functional diversity (Varshney et al., 2005). Transcriptomic SSR markers exhibit high interspecific transferability, which can be harnessed as functional markers in various genetic studies. The transcriptomic data obtained by 454 sequencing provides an excellent source for mining and developing gene-associated markers. In total, 1041 SSRs were identified by SSR Locator in $650(42.26 \%)$ unigenes of 
H. verticillata. Hexa-nucleotide repeats $(77.33 \%)$ were the most abundant SSRs in the transcriptome followed by trinucleotide $(14.32 \%)$ and the remaining repeat motifs were mononucleotide $(0.38 \%)$, dinucleotide $(1.83 \%)$, tetranucleotide $(5.28 \%$ ) and pentanucleotide $(0.86 \%)$ (Fig. 6a, 6b). The dominant repeat motif in all the SSRs identified in this study are AAGCGA/TCGCTT $(33,3.17 \%)$, followed by AAAATC/GATTTT $(15,1.44 \%)$, CTC/GAG $(15,1.44 \%)$, CCG/CGG $(13,1.25 \%)$ and GGA/TCC $(12,1.15 \%)$ (Table S7). Further validation in relation to phenotypic trait will be important.

\section{Materials and Methods}

\section{Plant growth conditions}

$H$. verticillata plants were collected from the Department of Botany, University of Delhi, New Delhi. The growth conditions were based on a modified version of Ginkel et al., (2001) and Rao et al., (2002) with changes in temperature. $H$. verticillata plants were kept in plastic tubs with irradiance of $300 \mu \mathrm{mol} \mathrm{m} \mathrm{s}^{-2}$ at the National Phytotron Facility, IARI Campus, New Delhi at $34{ }^{\circ} \mathrm{C} / 22^{\circ} \mathrm{C}$ for $14 / 10 \mathrm{hr}$ photoperiod's day/night to induce $\mathrm{C}_{4} \mathrm{CCM}(\mathrm{HvT})$ and at $20^{\circ} \mathrm{C} / 15^{\circ} \mathrm{C} 14 / 10$ hr to maintain $\mathrm{C}_{3}$ state $(\mathrm{HvC})$. Apical shoots with 40-45 leaves were harvested every day from the plants maintained under both conditions. These harvested shoots were flashfrozen in liquid $\mathrm{N}_{2}$ and stored at $-80^{\circ} \mathrm{C}$ till further use.

\section{RNA isolation and expression analysis of PEPC 2}

Total RNA was isolated from100mg plant material, HvT and $\mathrm{HvC}$, using the RaFlex ${ }^{\mathrm{TM}}$ Total RNA Isolation $\mathrm{Kit}\left(\mathrm{GeNe}^{\mathrm{TM}}\right.$, Bangalore, India) as per the manufacturer's protocol. Contaminating genomic DNA was removed by DNase I treatment (Qiagen, Hilden, Germany). The quality of the total RNA was checked on a $1.2 \%$ formaldehyde agarose gel under denaturing conditions.

Complementary DNA (cDNA) was prepared using the Superscript ${ }^{\circledR}$ III First Strand cDNA Synthesis Kit (Invitrogen, Carlsbad, CA) as per the manufacturer's protocol. Semiquantitative RT-PCR and Real-time PCR (qPCR) were performed to analyse the expression of $P E P C 2$ gene using gene specific primer and the $18 \mathrm{~S}$ rRNA primer as an internal control (Table S1).

\section{Preparation of cDNA library and transcriptome sequencing}

Messenger RNA (mRNA) was isolated from the total RNA of the samples ( $\mathrm{HvC}$ and $\mathrm{HvT}$; shifting to $\mathrm{C}_{4}$ state) using the Oligote ${ }^{\circledR}$ mRNA Midi Kit (Qiagen, Hilden, Germany) as per the manufacturer's protocol. The quality and quantity of RNA was determined using a Nanodrop 1000 (NanoDrop Technologies, USA) and a Bioanalyzer Chip RNA7500 series II (Agilent Technologies, USA). Equal quantities of high-quality mRNA from each sample ( $\mathrm{HvC}$ and $\mathrm{HvT}$ ) were used for the transcriptome study.

$\mathrm{HvC}$ and HvT cDNA were synthesized from respective mRNA using a cDNA Synthesis System (Roche, Basel, Switzerland) as per the manufacturer's protocol using hexamer primers (Roche, Basel, Switzerland). The cDNA samples were sheared by nebulisation to produce random fragments of approximately $300-800 \mathrm{bp}$ in length. The nebulised cDNA was recovered with the QIAquick ${ }^{\circledR}$ PCR Purification Kit (Qiagen, Hilden, Germany). Rapid Library Molecular Identifier (RL-MID) were ligated to the fragmented purified cDNA samples according to the standard procedure. One MID contains a barcode sequence that is used to discriminate between samples from different libraries. Library quantitation was performed using the TBS 380 Fluorometer (Turner Biosystems, CA, USA). The relative fluorescence unit of each dilution was recorded and checked against the RL standard curve, and the sample concentration was calculated. An Agilent Bioanalyzer High Sensitivity DNA chip was used to check the quality of the libraries. The quality-passed samples were pooled and amplified by emulsion PCR. The sequencing of the libraries was performed on a 454-GS FLX Titanium sequencer as per the manufacturer's protocol (454 Life Sciences, Roche, Basel, Switzerland).

\section{Sequencing data analysis and assembly}

All the obtained raw reads were processed to remove sequences smaller than 60 bases (including adapters and primer) based on the assumption that these small reads might represent sequencing artefacts. At the same time Q20, Q30, $\mathrm{GC}$ content and sequence duplication level of the clean data were collected. High quality reads were assembled into unique putative transcripts (including contigs and singletons) using the Newbler ESTs assembler (V 2.6). The assembly was performed using the default parameters.

\section{Unigene function annotation, $\mathrm{GO}$ classification and metabolic pathway analysis}

Blast homology searches and sequence annotations were carried out using Blast2GO software v.2.7.1 (http://www.blast2 go.org) (Conesa et al., 2005; Conesa and Gotz, 2008; Gotz et al., 2008). The assembled sequences were compared against the NCBI nr protein database via BLAST $X$ using an E-value cut-off of $1.0 \mathrm{E}^{-3}$. The searches were limited to the first 10 significant hits for each query to increase the computational speed. For gene ontology mapping (GO; http://www.geneontology.org), the program extracts the GO terms associated with homologies identified with NCBI's QBLAST and shows a list of GO annotations represented as hierarchical categories of increasing specificity. GO terms were modulated using the annotation augmentation tool, ANNEX (Myhre et al., 2006), followed by GO Slim (Harris et al., 2004). The data presented herein represent a GO analysis at level 2, demonstrating general functional categories. Enzyme classification (EC) codes were generated from direct mapping of GO terms to the enzyme code equivalents. GO term enrichment analysis was performed using Blast2GO software. This enrichment analysis was performed to evaluate the enrichment of various GO categories for the unigenes having expression values 2fold or above in both the samples.

The unigenes sequences were also aligned to the COG database to predict and classify proteins. KEGG pathways were assigned to the unigene sequences using the singledirectional best hit (SBH) method on the online KEGG Automatic Annotation Server (KAAS) (http://www.genome. jp/kegg/kaas) (Moriya et al., 2006). All the assembled unigenes were analysed against the PlantTFcat online tool (http://plantgrn.noble.org/PlantTFcat) for identification of the transcription factor in the $H$. verticillata transcriptome.

\section{GC content analysis and Simple Sequence Repeat (SSR) discovery}

Emboss GeeCee tool (http://emboss.bioinformatics.nl/cgibin/emboss/geecee) was used to measure the GC content of 
the sequences and SSR markers. SSR markers were identified using SSR Locator V.1 software (da Maia et al., 2008). Mononucleotides repeats of more than 12, dinucleotide repeats of more than 6 , trinucleotide repeats of more than 4 , tetranucleotide repeats of more than 3 , pentanucleotide repeats of more than 3 and hexanucleotide repeats of more than 2 were utilised as search criteria for SSRs.

\section{Expression analysis}

Clean reads from each of the libraries were used for mapping the sequences of each unigene and to calculate the unigene expression according to the "RPKM" method (Mortazavi et al., 2008). The significances of differences in gene expression between the $\mathrm{C}_{3}$ and $\mathrm{C}_{4}$ photosynthesis pathways were estimated using DEGseq, an $\mathrm{R}$ package [56]. The threshold of the P-value in multiple tests was identified using the False discovery rate (FDR), and the unigenes were considered as differentially expressed when the FDR was less than 0.05 and the $\log$ ratio was greater than 1 (two-fold change) between samples (Benjamini and Hochberg, 1995).

\section{Conclusion}

To the best of our knowledge, this is the first report to study the transcriptome of $H$. verticillata. A total of 533,595 high quality transcriptomic reads were obtained. A significant number of putative metabolic pathways and functions associated with the unique sequences were identified. Moreover, 1,041 SSRs were predicted among the unigenes, which can be used for marker development. These SSRs can also be utilized in studies involving genetic linkage and quantitative trait loci analysis. Overall, our transcriptome analysis of $H$. verticillata provides a valuable resource that will lead to a better understanding of the transition from the $\mathrm{C}_{3}$ to $\mathrm{C}_{4}$ photosynthetic pathway. This will aid in future studies related to engineering $\mathrm{C}_{4}$ photosynthetic pathway in $\mathrm{C}_{3}$ crop plants that will perform better in the event of rise in temperature and $\mathrm{CO}_{2}$ level due to global warming. Further investigations will be necessary to fully elucidate the role of putative genes identified in this study, during transition of $\mathrm{C}_{3}$ to $\mathrm{C}_{4}$ photosynthetic pathway in $H$. verticillata.

\section{Acknowledgment}

We would like to thank the staff of NGS Sequencing Laboratory, ICAR-NRCPB, New Delhi, India for providing high throughput sequencing facility.

\section{Author's contribution}

EG and RSS carried out experiment, prepared cDNA library for Roche sequencing, sequencing run and expression analysis. AKM performed read generation, process of assembling, expression analysis. EG and AKS carried out homology searching, annotation, GO, COG, KEGG pathway analysis, SSR markers discovery. KK conceived the study, designed the experiment, associated with wet lab work and coordinated the study. EG, AKS and KK did result interpretation, analysis and integration of results, drafted and finalized the manuscript. KK checked the final manuscript. All authors have read and approved the manuscript.

\section{Competing interests}

The authors declare that they have no competing interests.

\section{References}

Benjamini Y, Hochberg Y (1995) Controlling the false discovery rate a practical and powerful approach to multiple testing. $\mathbf{J}$ Roy Stat Soc B1. 57: 289-300.

Bowes G, Salvucci ME (1989) Plasticity in the photosynthetic carbon metabolism of submersed aquatic macrophytes. Aquat Bot. 34: 233-266.

Brandner SJC, Salvucci ME (2000) Rubisco activase constrains the photosynthetic potential of leaves at high temperature and $\mathrm{CO}_{2}$. P Natl Acad Sci USA. 97: 13430-13435.

Brautigam A, Gowik U (2010) What can next generation sequencing do for you? Next generation sequencing as a valuable tool in plant research. Plant Biol. 12: 831-841.

Brautigam A, Kajala K, Wullenweber J, Sommer M, Gagneul D et al (2011) An mRNA blueprint for $\mathrm{C}_{4}$ photosynthesis derived from comparative transcriptomics of closely related $\mathrm{C}_{3}$ and $\mathrm{C}_{4}$ species. Plant Physiol. 155: 142-156.

Brautigam A, Schliesky S, Kulahoglu C, Osborne CP, Weber AP (2014) Towards an integrative model of $\mathrm{C}_{4}$ photosynthetic subtypes insights from comparative transcriptome analysis of NAD-ME, NADP-ME and PEP-CK C 4 species. J Exp Bot. 65: 3579-3593.

Conesa A, Gotz S (2008) Blast2GO: A comprehensive suite for functional analysis in plant genomics. Int J Plant Genomics. 2008: 619832.

Conesa A, Gotz S, Garcia-Gomez JM, Terol J, Talon M, et al (2005) Blast2GO a universal tool for annotation visualisation and analysis in functional genomics research. Bioinformatics. 21: 3674-3676.

da Maia LC, Palmieri DA, de Souza VQ, Kopp MM, de CarvalhoFIF, et al (2008) SSR Locator Tool for Simple Sequence Repeat Discovery Integrated with Primer Design and PCR Simulation. Int J Plant Genomics. 4: 363-374.

Edwards GE, Franceschi VR, Voznesenskaya EV (2004) Singlecell $\mathrm{C}_{4}$ photosynthesis versus the dual-cell (Kranz) paradigm. Annu Rev Plant Biol. 55: 173-196.

Estavillo GM, Rao SK, Reiskind JB, Bowes G (2007) Characterisation of the NADP malic enzyme gene family in the facultative single-cell $\mathrm{C}_{4}$ monocot Hydrilla verticillata. Photosynth Res. 94: 43-57.

Flicek P, Birney E (2009) Sense from sequence reads methods for alignment and assembly. Nat Methods. 6: 6-12.

Freitag H, Stichler W (2000) A remarkable new leaf type with unusual photosynthetic tissue in a central asiatic genus of chenopodiaceae. Plant Biol. 2: 154-160.

Furbank RT, von Caemmerer S, Sheehy J, Edwards G (2009) $\mathrm{C}_{4}$ rice a challenge for plant phenomics. Funct Plant Biol. 36: 845-856.

Ginkel LC, Bowes G, Reiskind JB, Prins HBA (2001) $\mathrm{A} \mathrm{CO}_{2-}$ flux mechanism operating via $\mathrm{pH}$-polarity in Hydrilla verticillata leaves with $\mathrm{C}_{3}$ and $\mathrm{C}_{4}$ photosynthesis. Photosynth Res. 68: 81-88.

Gotz S, Garcia-Gomez JM, Terol J, Williams TD, Nagaraj SH (2008) High-throughput functional annotation and data mining with the Blast2GO suite. Nucleic Acids Res. 36: 3420-3435.

Gowik U, Westhoff $\mathrm{P}$ (2011) The path from $\mathrm{C}_{3}$ to $\mathrm{C}_{4}$ photosynthesis. Plant Physiol. 155: 56-63.

Guo ZJ, Chen XJ, Wu XL, Ling JQ, Xu P (2004) Overexpression of the AP2/EREBP transcription factor OPBP1 enhances disease resistance and salt tolerance in tobacco. Plant $\mathrm{Mol}$ Biol. 55: 607-618.

Harris MA, Clark J, Ireland A, Lomax J, Ashburner M, et al (2004) The gene ontology (GO) database and informatics resource. Nucleic Acids Res. 32: 258-261.

Hatch MD (1988) $C_{4}$ photosynthesis a unique blend of modified biochemistry anatomy and ultrastructure. Biochim Biophys Acta. 895: 81-106. 
Hausler RE, Hirsch HJ, Kreuzaler F, Peterhansel C (2002) Overexpression of $\mathrm{C}_{4}$-cycle enzymes in transgenic $\mathrm{C}_{3}$ plants a biotechnological approach to improve $\mathrm{C}_{3}$-photosynthesis. $\mathrm{J}$ Exp Bot. 53: 591-607.

Hsieh TH, Lee JT, Yang PT, et al (2002) Heterology expression of the Arabidopsis C-repeat/dehydration response element binding factor 1 gene confers elevated tolerance to chilling and oxidative stresses in transgenic tomato. Plant Physiol. 129: 1086-1094.

Kim JC, Lee SH, Cheong YH, et al (2001) A novel coldinducible zinc finger protein from soybean SCOF-1 enhances cold tolerance in transgenic plants. Plant J. 25: 247-259.

Kobza J, Edwards GE (1987) Influences of leaf temperature on photosynthetic carbon in metabolism in wheat. Plant Physiol. 83: 69-74.

Kuhl JC, Cheung F, Yuan Q, Martin W, Zewdie Y, et al (2004) A unique set of 11008 onion expressed sequence tags reveals expressed sequence and genomic differences between the monocot orders asparagales and poales. Plant Cell. 16: 114125.

Li W, Godzik A (2006) Cd-hit a fast program for clustering and comparing large sets of protein or nucleotide sequences. Bioinformatics. 22: 1658-1659.

Magnin NC, Cooley BA, Reiskind JB, et al (1997) Regulation and localization of key enzymes during the induction of Kranzless $\mathrm{C}_{4}$ photosynthesis in Hydrilla verticillata. Plant Physiol. 115: 1681-1689.

Matsuoka M, Furbank RT, Fukayama H, Miyao M (2001) Molecular engineering of $\mathrm{C}_{4}$ photosynthesis. Annu Rev Plant Phys. 52: 297-314.

Metzker ML (2010) Applications of next-generation sequencing technologies the next generation. Nat Rev Genet. 11: 31-46.

Miyao M (2003) Molecular evolution and genetic engineering of $\mathrm{C}_{4}$ photosynthetic enzymes. J Exp Bot. 54: 179-189.

Miyao M, Masumoto C, Miyazawa S, Fukayama H (2011) Lessons from engineering a single-cell $\mathrm{C}_{4}$ photosynthetic pathway into rice. J Exp Bot. 62: 3021-3029.

Miyoshi K, Ito Y, Serizawa A, Kurata N (2003) OsHAP3 genes regulate chloroplast biogenesis in rice. Plant J. 36: 532-540.

Moriya Y, Itoh M, Okuda S, Yoshizawa AC, Kanehisa M (2006) KAAS an automatic genome annotation and pathway reconstruction server. Nucleic Acids Res. 35: 182-185.

Mortazavi A, Williams BA, McCue K, Schaeffer L, Wold B (2008) Mapping and quantifying mammalian transcriptomes by RNA-Seq. Nat Methods. 5: 621-628.

Myhre S, Tveit H, Mollestad T, Laegreid A (2006) Additional gene ontology structure for improved biological reasoning. Bioinformatics. 22: 2020-2027.

Oaks A (1994) Efficiency of nitrogen utilization in $\mathrm{C}_{3}$ and $\mathrm{C}_{4}$ cereals. Plant Physiol. 106: 407-414.

Oh SJ, Kwon CW, Choi DW, Song SI, Kim JK (2007) Expression of barley HvCBF4 enhances tolerance to abiotic stress in transgenic rice. Plant Biotechnol J. 5: 646-656.

Park JM, Park CJ, Lee SB, Ham BK, Shin R, Paek KH (2001) Overexpression of the tobacco Tsil gene encoding an EREBP/AP2-type transcription factor enhances resistance against pathogen attack and osmotic stress in tobacco. Plant Cell. 13: 1035-1046.

Powell W, Machray GC, Provan J (1996) Polymorphism revealed by simple sequence repeats. Trends Plant Sci. 1: 215222.

Rao SK, Fukayama H, Reiskind J, Miyao M, Bowes G (2006a). Identification of $\mathrm{C}_{4}$ responsive genes in the facultative $\mathrm{C}_{4}$ plant Hydrilla verticillata. Photosynth Res. 88: 173-183

Rao SK, Magnin NC, Reiskind JB, Bowes G (2002) Photosynthetic and other phosphoenolpyruvate carboxylase isoforms in the single cell facultative $\mathrm{C}_{4}$ system of Hydrilla verticillata. Plant Physiol. 130: 876-886.

Rao SK, Reiskind JB, Bowes G (2006b) Light regulation of the photosynthetic phosphoenolpyruvate carboxylase (PEPC) in Hydrilla verticillata. Plant Cell Physiol. 47: 1206-1216.
Rao SK, Reiskind JB, Bowes G (2008) Kinetic analyses of recombinant isoforms of phosphoenolpyruvate carboxylase from Hydrilla verticillata leaves and the impact of substituting a $\mathrm{C}_{4}$-signature serine. Plant Sci. 174: 475-483.

Reiskind JB, Berg RH, Salvucci ME, Bowes G (1989) Immunogold localization of primary carboxylases in leaves of aquatic and a $\mathrm{C}_{3} \mathrm{C}_{4}$ intermediate species. Plant Sci. 61: 43-52.

Reiskind JB, Maberly SC (2014) A tribute to george bowes linking terrestrial and aquatic botany. Aquat Bot. 118: 1-3.

Reiskind JB, Madsen TV, Van Ginkel LC, Bowes G (1997) Evidence that inducible $\mathrm{C}_{4}$-type photosynthesis is a chloroplastic $\mathrm{CO}_{2}$-concentrating mechanism in Hydrilla a submersed monocot. Plant Cell Environ. 20: 211-220.

Reynolds M, Bonnett D, Chapman SC, Furbank RT, Mane Y, Mather DE, Parry MAJ (2010) Raising yield potential of wheat. I. overview of a consortium approach and breeding strategies. J Exp Bot. 62: 439-52.

Sage RF (1990) A model describing the regulation of ribulose-15-bisphosphate carboxylase electron transport and triose phosphate use in response to light intensity and $\mathrm{CO}_{2}$ in $\mathrm{C}_{3}$ plants. Plant Physiol. 94: 1728-1734.

Saibo NJM, Lourenco T, Oliveira MM (2009) Transcription factors and regulation of photosynthetic and related metabolism under environmental stresses. Ann Bot-London. 103: 609-623

Sheehy JE, Mitchell PL, Hardy B (2000) Redesigning Rice Photosynthesis to Increase Yield. Proceedings of the Workshop on the Quest to Reduce Hunger: Redesigning Rice Photosynthesis, 30 November - 3 December 1999, Los Baños, Philippines. Makati City (Philippines): International Rice Research Institute and Amsterdam (The Netherlands): Elsevier Science B.V. pp-293.

Tatusov RL, Galperin MY, Natale DA, Koonin EV (2000) The COG database a tool for genome-scale analysis of protein functions and evolution. Nucleic Acids Res. 28: 33-36.

Varshney RK, Graner A, Sorrells ME (2005) Genic microsatellite markers in plants features and applications. Trends Biotechnol. 23: 48-55.

Voznesenskaya EV, Franceschi VR, Kiirats O, Freitag $\mathrm{H}$, Edwards GE (2001) Kranz anatomy is not essential for terrestrial $\mathrm{C}_{4}$ plant photosynthesis. Nature. 414: 543-546.

$\mathrm{Vu}$ JCV, Allen LH, Boote KJ, Bowes Gb (1997) Effects of elevated $\mathrm{CO}_{2}$ and temperature on photosynthesis and rubisco in rice and soybean. Plant Cell Environ. 20: 68-76.

Wang L, Feng Z, Wang X, Wang X, Zhang X (2010) DEGseq an $\mathrm{R}$ package for identifying differentially expressed genes from RNA-seq data. Bioinformatics. 26: 136-138.

Wei W, Qi X, Wang L, Zhang Y, Hua W, Li D, Lv H, Zhang X (2011) Characterization of the sesame (Sesamum indicum L) global transcriptome using Illumina paired-end sequencing and development of EST-SSR markers. BMC Genomics. 12: 451464

Zhang X, Wollenweber B, Jiang D, Liu F, Zhao J (2008) Water deficits and heat shock effects on photosynthesis of a transgenic Arabidopsis thaliana constitutively expressing ABP9 a bZIP transcription factor. J Exp Bot. 59: 839-848. 